\title{
Enhancing spontaneous stem cell healing (Review)
}

\author{
GREG MAGUIRE and PETER FRIEDMAN \\ BioRegenerative Sciences, Inc., San Diego, CA 92014, USA \\ Received October 18, 2013; Accepted October 21, 2013
}

DOI: $10.3892 /$ br.2014.231

\begin{abstract}
Adult stem cells are distributed throughout the human body and are responsible to a great extent for the body's ability to maintain and heal itself. Accumulating data since the 1990s regarding stem cells have demonstrated that the beneficial effects of stem cells are not restricted to their ability to differentiate and are more likely due to their ability to release a multitude of molecules. Recent studies indicated that $\leq 80 \%$ of the therapeutic benefit of adult stem cells is manifested by the stem cell released molecules (SRM) rather than the differentiation of the stem cells into mature tissue. Stem cells may release potent combinations of factors that modulate the molecular composition of the cellular milieu to evoke a multitude of responses from neighboring cells. A multitude of pathways are involved in cellular and tissue function and, when the body is in a state of disease or trauma, a multitude of pathways are involved in the underlying mechanisms of that disease or trauma. Therefore, stem cells represent a natural systems-based biological factory for the production and release of a multitude of molecules that interact with the system of biomolecular circuits underlying disease or tissue damage. Currently, efforts are aimed at defining, stimulating, enhancing and harnessing SRM mechanisms, in order to develop systems-based methods for tissue regeneration, develop drugs/biologics or other therapeutics and enhance the release of SRM into the body for natural healing through proper dietary, exercise and other lifestyle strategies.
\end{abstract}

\section{Contents}

1. Introduction

2. Multiple stem cell types in healing

3. A multitude of molecules in healing

4. Slowing the aging process of stem cells

5. Conclusions

Correspondence to: Dr Greg Maguire, BioRegenerative Sciences, Inc., SRM Molecular Foundry, 2658 Del Mar Heights Road, Suite 416, San Diego, CA 92014, USA

E-mail: gmaguire@bioregenerativesciences.com

Key words: adult stem cell, healing, autophagy, paracrine, autocrine, juxtacrine

\section{Introduction}

In the post-genomic era, it is well-known that the phenotypic expression of normal and disease states is rarely predicted by the genome (1) and the genetic make-up of somatic cells may be altered during the lifespan of an individual (2). These phenotypic states reflect a complex set of interactions between the genome and numerous variables, including diet, lifestyle, epigenetics, toxins, non-genetic inheritable transmission and environment (3). Several of the disease phenotypes are currently considered to involve the underlying state of adult stem cells, which are located throughout the body and are involved in the maintenance, regeneration and repair of the majority, or all, human tissues. The pancreas may represent an exception, where the somatic cells themselves replicate (4). As regards aging, although the effects are tissue-dependent, certain tissues exhibit a decline in stem cell numbers during the aging process, while other tissues may exhibit an increase. However, it is well-known that aging is associated with a progressive decline in stem cell function in various tissues (5). This age-related decrease in stem cell function is partially a consequence of the number of times the stem cell replicates (6), although it is also considered to be associated with intrinsic and environmental changes in the stem cell niche (7), such as a decrease of the self-renewal molecule Upd within the stem cell niche, leading to a lower number of active stem cells (8). Thus, during aging, as the environment deviates from the ideal, the gradual decline in stem cell function may lead to an impaired ability of self-healing and may be directly associated with certain diseases $(6,9)$.

\section{Multiple stem cell types in healing}

Multiple adult stem cell types are located in the majority of tissues in adult humans and reside in stem cell niches within various tissues, in either a dormant or an active state (10). The pool of active stem cells is readily available for tissue repair, regeneration and maintenance. To heal and maintain the various tissues of the body, including the skin (11), multiple stem cell types are often involved (12) and secrete a number of factors into the tissue to induce the healing response (13-15). Thus, stem cells in the human body may migrate to the area of diseased or damaged tissue and two or more stem cell types release their respective stem cell released molecules (SRM) into the damaged tissue (Fig. 1). Each stem cell type releases its own pool of SRM, each pool being a different collection of molecules, with a certain degree of overlap between pools. 


\section{A multitude of molecules in healing}

The release of factors from stem cells includes several molecules, such as cytokines, growth factors, interleukins, antioxidants, chaperone molecules, exosomes and microRNAs (15). The factors released from stem cells are collectively referred to as SRM, and when the SRM comes from two or more types of stem cells, the collection of molecules is called $\mathrm{S}^{2} \mathrm{RM}$, reflecting the synergistic action of the two pools of SRM on the target tissue. The release of SRM is a key method for tissue maintenance, repair and regeneration, including immune modulation functions (15), whereas asymmetric regeneration of adult cells, with some of the replicated stem cells differentiating into mature cells, may be another method for tissue healing and maintainance (16).

\section{Slowing the aging process of stem cells}

If the human genome is not predictive of health and disease and human life is not predetermined through genetics, we must identify the factors that may be controlled in order to maintain a healthy state and enhance spontaneous stem cell healing. First, the data reported by Toledano et al (8) suggested that simply engrafting new or young stem cells into an old environment, such as the stem cell niche of an aged patient, may not lead to the optimal outcome regarding tissue regeneration and age-related conditions. Therefore, the stem cells themselves must be in an active state; however, the cells of the stem cell niche, other than the actual stem cells, must also be in a healthy and 'active' state and able to provide the proper signaling environment to maintain stem cell activity (17).

Telomeres in stem cells, similar to other cell types, are specialized chromatin structures at the ends of eukaryotic chromosomes that prevent the chromosome ends from being recognized as a DNA break. Although the pathways by which short telomeres promote aging have not been fully elucidated, telomere shortening may promote aging by inducing apoptosis and cell cycle arrest, thus leading to cell loss and tissue dysfunction. Furthermore, telomere shortening may impair the ability of stem cells to regenerate tissues, thus leading to tissue failure. It is well-known that shortened telomeres in stem cells impede their mobilization out of the niche, inhibit tissue repair and regeneration and suppress stem cell proliferation (18). Furthermore, stem cell telomeres are shortened with progressing age (18).

Previous studies indicated that exercise, lifestyle and diet may lengthen telomeres and improve stem cell function. Exercise was shown to lengthen or preserve human telomere length $(19,20)$ and combat the effects of stress on telomere length (21). Furthermore, Kadi and Ponsot (22) demonstrated that telomeres in skeletal muscle are dynamic structures affected by their environment. When satellite cells were heavily recruited for regenerative events, as in the skeletal muscle of athletes, telomere length was found to be either significantly shortened or maintained, and was even found to be longer compared to that in non-trained individuals. Evidence suggested that physical activity may preserve or lengthen telomeres, although exhaustive exercise, such as that performed by athletes, may shorten the telomeres in skeletal muscle (23). Moreover, exercise induces autophagy (24), thus clearing the
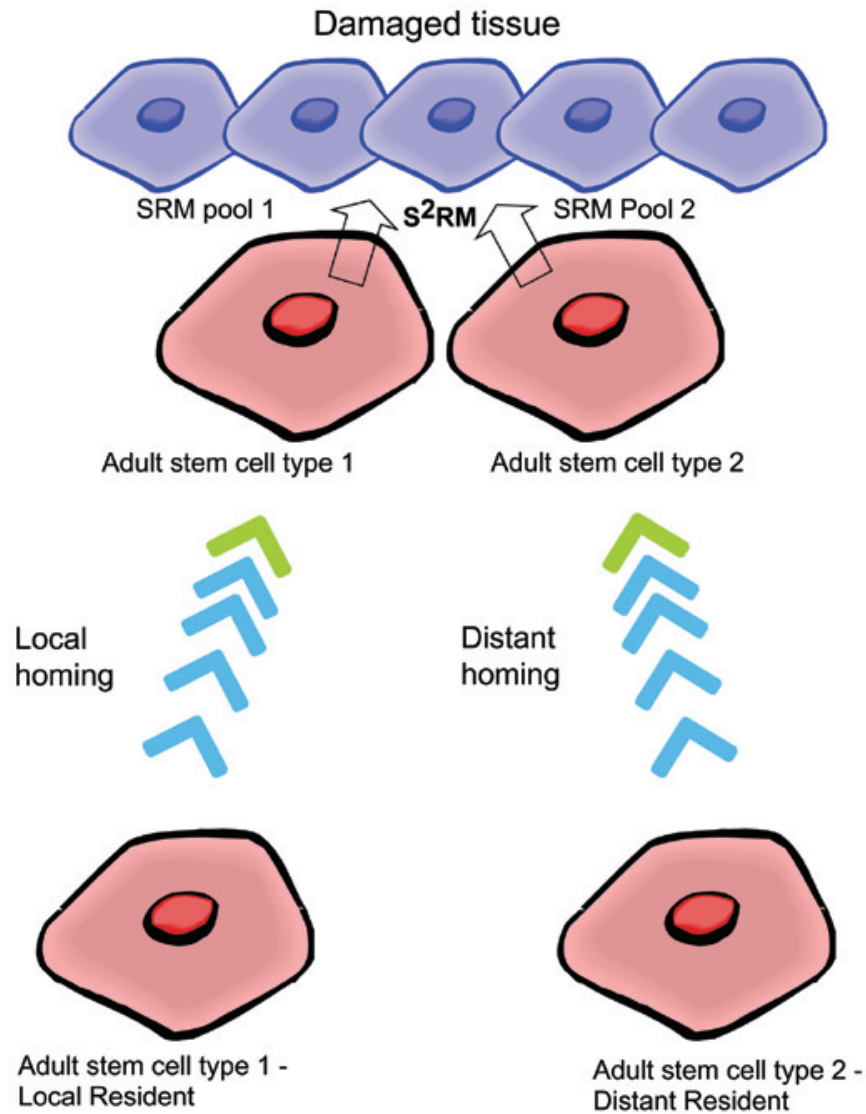

Figure 1. Two stem cell types home into the damaged tissue and release stem cell released molecules (SRM) to induce healing. The SRM from stem cell type 1 is different from that of type 2 . The two SRM pools act synergistically and are referred to as $\mathrm{S}^{2} \mathrm{RM}$.

\section{Stem cells in niche}

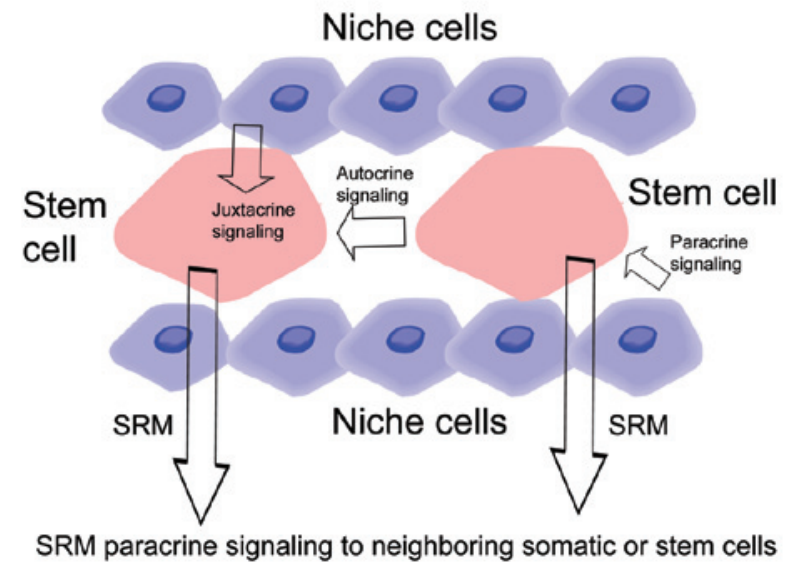

Figure 2. Adult stem cells exist within niches, where a rich interaction occurs between the stem cells and other niche cells, such as endothelial cells and adipocytes. SRM, stem cell released molecules.

debris from the stem cell niche and allowing the stem cells and the niche to properly interact and, therefore, enhance stem cell function. Valero et al (25) demonstrated that stem cells release SRM during exercise. Maguire and Friedman $(15,26)$ 
also demonstrated that SRM may exert multiple effects on the body, including pain reduction, tissue regeneration, immune system modulation and possible telomere lengthening. Those data suggested that exercise may induce several useful mechanisms that may optimize stem cell function and enable the highly beneficial release of SRM and a multitude of beneficial downstream physiological effects.

Recent data from the Kipnis Lab (27) also demonstrated that the immune system regulates the neurogenic stem cell niche, suggesting that those factors boosting immune function may also boost stem cell-based self renewal. Fig. 2 illustrates some of the key signaling pathways in the stem cell niche. It is crucial to identify the factors that boost immune system function, allowing the proper function of the neurogenic niche, or even of all stem cell niches throughout the body. The answer again lies with exercise, diet and lifestyle. These lifestyle changes may induce autophagy, so that the niche is cleared of debris, thus optimizing stem cell activation and migration from the niche to the damaged tissue. The lifestyle changes may also enhance SRM-mediated paracrine and autocrine signaling and, at least in some stem cell types, is likely to increase juxtacrine signaling (28).

Calorie restriction (CR) may extend the life span and ameliorate age-related pathologies in the majority of investigated species; however, the mechanisms underlying these effects have not been fully elucidated. A number of studies demonstrated that $\mathrm{CR}$ acts in part by enhancing the function of tissue-specific stem cells (29). Even a short-term CR may significantly enhance stem cell availability and activity, as observed in muscle tissue (30).

Paneth cells, a key constituent of the intestinal stem cell (ISC) niche, which reside adjacent to stem cells, may augment stem cell function in response to $\mathrm{CR}$. CR acts by reducing mechanistic target of rapamycin complex 1 signaling in Paneth cells and the ISC-enhancing effects of CR may be mimicked by rapamycin (29). In another study, CR was shown to enhance adult stem cell availability and function in muscle, improving the efficacy of recovery from injury and the engraftment of transplanted cells (30). Those studies indicated that metabolic factors are crucial in the regulation of stem cell function and that this regulation may improve the organism's natural spontaneous stem cell healing. The restriction of calorie ingestion, without malnutrition, prolongs lifespan and promotes healthy aging in numerous animal species and may be at least partly attributed to the enhanced stem cell function. Campbell and Campbell (31) reported sound evidence supporting the reduction of protein intake to $\sim 10 \%$ of the caloric intake in order to increase lifespan and reduce the risk of disease. Furthermore, Fontana et al (32) demonstrated that reduced protein intake reduces the risk of cancer. However, the effect of specific dietary restrictions has not been clearly determined regarding stem cells.

Stress. Stress exerts a number of debilitating effects on health. As demonstrated by a recent animal model of cancer and cancer survival, Rottweilers are prone to cancer and, despite a body riddled with cancerous growths, a certain percentage of Rottweilers live to an old age and die due to causes other than cancer. Furthermore, cancer in these animals tends to develop in distinct regions of the body (33). A recent study by Waters (33) demonstrated that the senior animals expressed little stress, as revealed by their behavior. Furthermore, the animals exhibiting little behavioral stress also exhibited low levels of cortisol. Low blood levels of cortisol would not have been predicted in these aged dogs based on the literature. Although exhibiting low levels of cortisol, those animals were able to increase their cortisol levels when challenged, which reflects normal adrenal function.

Tumors are characterized by the disordered structure of extracellular matrix (ECM) and unregulated cell growth. Cells present within the tumor interact, not only in an autocrine or paracrine manner, but also with the ECM components. The ECM is a dynamic niche involved in cancer progression (34) and adult stem cells secrete a number of matrix molecules that appear to be important for tissue growth regulation. It is well-known that the stress-induced secretion of cortisol disrupts the ECM (35) and that the SRM may mitigate the effects of stress (36). Furthermore, in the stress pathway, stem cells are inhibited from being activated and released into the damaged tissue $(37,38)$. An important component of the response to stress, the autonomic nervous system, appears to directly or indirectly mediate stem cell function in the stromal tissues in several parts of the body and regulate cancer growth and dissemination (39). Thus, stress likely affects a number of pathways throughout the body, deregulating the ECM. Therefore, stress may be an important contributor to cancer development and progression, as well as other conditions, including disruption of epidermal function (40).

\section{Conclusions}

Reverse-engineering the endogenous stem cell mechanisms and their control mechanisms underlying tissue maintenance and healing may be instructive for an understanding of lifestyle and therapeutic development that may enhance the maintenance and healing functions of the human body. Traditional drug development is based on the investigation of chromatin regulators and transcription factors that may affect stem cell maintenance, particularly in tumor development, and the evidence indicates that such manipulations of adult stem cell function in aged individuals must be performed with caution, considering the increased risk of cellular transformation. However, previous studies on the effect of exercise, environmental enrichment, SRM and parabiosis on aged stem cells revealed that rejuvenation of stem cells may be achieved without the induction of neoplastic properties.

Elucidating the synergistic or antagonistic roles of different chromatin and stem cell niche regulators, such as SRM and $\mathrm{S}^{2} \mathrm{RM}$, and their primary targets, as well as the external signaling pathways responsible for that regulation, is crucial for restoring the regenerative potential of aging adult stem cells and their niches in a controlled manner. The enhancement of the regenerative potential of dormant endogenous stem cells and their SRM may be a promising avenue of prevention and treatment of several age-dependent and immune diseases and other types of trauma that are characterized by tissue degeneration, leading to enhanced spontaneous stem cell healing and resulting tissue healing and regeneration, as envisioned by such early pioneers as the famous aviator and biomedical engineer Charles Lindbergh and his partner, Nobel Laureate Alexis Carrel (41). 


\section{References}

1. Maguire G, Lee P, Manheim D and Boros L: SiDMAP: a metabolomics approach to assess the effects of drug candidates on the dynamic properties of biochemical pathways. Expert Opin Drug Discov 1: 351-359, 2006.

2. Baillie JK, Barnett MW, Upton KR, et al: Somatic retrotransposition alters the genetic landscape of the human brain. Nature 479 534-537, 2011.

3. Maguire G: Using a systems-based approach for the development of diagnostics. Expert Rev Mol Diagn (In press).

4. Stanger BZ and Hebrok M: Control of cell identity in pancreas development and regeneration. Gastroenterology 144: 1170-1179, 2013.

5. Jones DL and Rando TA: Emerging models and paradigms for stem cell ageing. Nat Cell Biol 13: 506-512, 2011.

6. Sharpless NE and DePinho RA: How stem cells age and why this makes us grow old. Nat Rev Mol Cell Biol 8: 703-713, 2007.

7. Waterstrat A and Van Zant G: Effects of aging on hematopoietic stem and progenitor cells. Curr Opin Immunol 21: 408-413, 2009.

8. Toledano H, D'Alterio C, Czech B, Levine E and Jones DL: The let-7-Imp axis regulates ageing of the Drosophila testis stem-cell niche. Nature 485: 605-610, 2012.

9. Xu Q, Seeger FH, Castillo J, et al: Micro-RNA-34a contributes to the impaired function of bone marrow-derived mononuclear cells from patients with cardiovascular disease. J Am Coll Cardiol 59: 2107-2117, 2012.

10. Li L and Clevers H: Coexistence of quiescent and active adult stem cells in mammals. Science 327: 542-545, 2010.

11. Yan X and Owens DM: The skin: a home to multiple classes of epithelial progenitor cells. Stem Cell Rev 4: 113-118, 2008

12. Guo S and Dipietro LA: Factors affecting wound healing. J Dent Res 89: 219-229, 2010.

13. Lu P, Jones LL, Snyder EY and Tuszynski MH: Neural stem cells constitutively secrete neurotrophic factors and promote extensive host axonal growth after spinal cord injury. Exp Neurol 181: $115-129,2003$.

14. Teng YD, Yu D, Ropper AE, et al: Functional multipotency of stem cells: a conceptual review of neurotrophic factor-based evidence and its role in translational research. Curr Neuropharmacol 9: 574-585, 2011.

15. Maguire G and Friedman P: The systems biology of stem cell released molecules-based therapeutics. ISRN Stem Cells 2013: $784541,2013$.

16. Clarke DL, Johansson CB, Wilbertz J, et al: Generalized potential of adult neural stem cells. Science 288: 1660-1663, 2000

17. Scadden DT: The stem-cell niche as an entity of action. Nature 441: 1075-1079, 2011

18. Flores I, Cayuela ML and Blasco MA: Effects of telomerase and telomere length on epidermal stem cell behavior. Science 309: 1253-1256, 2005.

19. LaRocca TJ, Seals DR and Pierce GL: Leukocyte telomere length is preserved with aging in endurance exercise-trained adults and related to maximal aerobic capacity. Mech Ageing Dev 131: 165-167, 2010.

20. Werner C, Furster T, Roggia C, et al: Beneficial effects of long-term endurance exercise on leukocyte telomere biology. Circulation 120: S492, 2009.

21. Puterman E, Lin J, Blackburn E, et al: The power of exercise: buffering the effect of chronic stress on telomere length. PLoS One 5: e10837, 2010.
22. Kadi F and Ponsot E: The biology of satellite cells and telomeres in human skeletal muscle: effects of aging and physical activity. Scand J Med Sci Sports 20: 39-48, 2010.

23. Collins M, Renault V, Grobler LA, et al: Athletes with exerciseassociated fatigue have abnormally short muscle DNA telomeres. Med Sci Sports Exerc 35: 1524-1528, 2003.

24. He C, Bassik MC, Moresi V, et al: Exercise-induced BCL2regulated autophagy is required for muscle glucose homeostasis. Nature 481: 511-515, 2012.

25. Valero MC, Huntsman HD, Liu J, Zou K and Boppart MD: Eccentric exercise facilitates mesenchymal stem cell appearance in skeletal muscle. PLoS One 7: e29760, 2012.

26. Maguire G and Friedman P: Stem cell-based systems biology approach for developing therapeutics to manage peripheral pain. Society for Neuroscience Annual Meeting Abstracts, 2011.

27. Lu Z, Elliott MR, Chen Y, et al: Phagocytic activity of neuronal progenitors regulates adult neurogenesis. Nat Cell Biol 13: 1076-1083, 2011

28. Govey PM, Loiselle AE and Donahue HJ: Biophysical regulation of stem cell differentiation. Curr Osteoporos Rep 11: 83-91, 2013.

29. Yilmaz OH, Katajisto P, Lamming DW, et al: mTORC1 in the Paneth cell niche couples intestinal stem-cell function to calorie intake. Nature 486: 490-495, 2012.

30. Cerletti M, Jang YC, Finley LW, et al: Short-term calorie restriction enhances skeletal muscle stem cell function. Cell Stem Cell 10: 515-519, 2012.

31. Campbell TC and Campbell TM II (eds): The China Study: Startling Implications for Diet, Weight Loss, and Long-term Health. BenBella Books, Dallas, TX, pp, 2005.

32. Fontana L, Klein S and Holloszy JO: Long-term low-protein, low-calorie diet and endurance exercise modulate metabolic factors associated with cancer risk. Am J Clin Nutr 84: 1456-1462, 2006.

33. Waters DJ: Aging research 2011: exploring the pet dog paradigm. ILAR J 52: 97-105, 2011.

34. Lu P, Weaver VM and Werb Z: The extracellular matrix: a dynamic niche in cancer progression. J Cell Biol 196: 395-406, 2012.

35. Houck JC, Sharma VK, Patel YM and Gladner JA: Induction of collagenolytic and proteolytic activities by anti-inflammatory drugs in the skin and fibroblast. Biochem Pharmacol 17: 2081-2090, 1968.

36. Zhou Y, Xu H, Xu W, et al: Exosomes released by human umbilical cord mesenchymal stem cells protect against cisplatininduced renal oxidative stress and apoptosis in vivo and in vitro. Stem Cell Res Ther 4: 34, 2013.

37. Katayama Y, Battista M, Kao WM, et al: Signals from the sympathetic nervous system regulate hematopoietic stem cell egress from bone marrow. Cell 124: 407-421, 2006.

38. Mendez-Ferrer S, Lucas D, Battista M and Frenette PS: Haematopoietic stem cell release is regulated by circadian oscillations. Nature 452: 442-447, 2008.

39. Magnon C, Hall SJ, Lin J, et al: Autonomic nerve development contributes to prostate cancer progression. Science 341: 1236361, 2013.

40. Garg A, Chren MM, Sands LP, et al: Psychological stress perturbs epidermal permeability barrier homeostasis: implications for the pathogenesis of stress-associated skin disorders. Arch Dermatol 137: 53-59, 2001.

41. Carrel A and Lindbergh CA (eds): The Culture of Organs. Paul B Hoeber, New York, NY, pp, 1938. 